\title{
Exploring the Barnyard Millet (Echinochloa frumentacea Roxb. Link) Segregating Population for Isolation of High Yielding, Iron and Zinc Content Genotype
}

\author{
V.G. Renganathan* and C. Vanniarajan
}

Department of Plant Breeding and Genetics, Agricultural College and Research Institute, Tamil Nadu Agricultural University, Madurai-625104, India

*Corresponding author

\begin{tabular}{|l|}
\hline Ke y w o r d s \\
$\begin{array}{l}\text { Barnyard millet, Parent } \\
\text { progeny regression, } \mathrm{F}_{2} \\
\text { and } \mathrm{F}_{3} \text { population, } \\
\text { Micronutrient }\end{array}$ \\
\hline Article Info \\
\hline $\begin{array}{l}\text { Accepted: } \\
\text { 30 March } 2018 \\
\text { Available Online: } \\
\text { 10 April 2018 }\end{array}$ \\
\hline
\end{tabular}

\section{Introduction}

Small millets are nutrient-rich food sources traditionally grown and consumed by subsistence farmers in Asia and Africa. They include finger millet, foxtail millet, proso millet, little millet, barnyard millet, kodo millet, teff and fonio. All these crops have an old history of cultivation, going behind $3000-$ 5000 years and probably were amongst the
A field study was carried out involving three $F_{2}$ and $F_{3}$ population of barnyard millet crosses during 2016-2017. The high PCV and GCV values and high heritability were noticed in $\mathrm{F}_{2}$ population of three crosses for number of tillers, iron and zinc content, indicated the predominance of additive gene effects in their expression and would respond to selection effectively as they are least influenced by environment. In the $\mathrm{F}_{3}$ generation, the identified superior families in all the three crosses revealed that mean performance of yield and yield component characters progressively increased when compared to control [Co (Kv) 2] except for days to maturity and number of racemes (ACM $331 \times$ ACM335, ACM 331 x ACM333) and single ear head weight (ACM 331 x ACM335). Three out of ten families of ACM 331 x MA10 cross exhibited superior mean performance than the control for most of the yield attributing and micronutrient traits. The coefficient of variation among the progenies of ACM 331 x MA10 cross was low indicated the settling down of homozygosity in $\mathrm{F}_{3}$ generation itself. These selected families are recommended for promoting to advanced generation for varietal development program with respect to high yield coupled with high iron and zinc content. The parent progeny correlation and regression between two generation $\left(\mathrm{F}_{2}\right.$ and $\left.\mathrm{F}_{3}\right)$ of all the three crosses showed their lesser susceptible to environmental effect (i.e.) consistence performance. 
soils (Sherif, 2007; Abe et al., 2011). It is also cultivated at high hills under double cut production system with increased yield (Bandyopadhyay, 2009). Barnyard millet being a promising source of micronutrients and protein besides energy, can make a contribution to alleviating micronutrient and protein malnutrition, also called 'hiddenhunger', affecting more than half of the world's population, especially women and pre-school children in most countries of Africa and south-east Asia (Underwood, 2000). The iron and zinc content in grains of barnyard millet ranged from 2.29 to $18.00 \mathrm{mg} / 100 \mathrm{~g}$ and 1.5 to $7.5 \mathrm{mg} / 100 \mathrm{~g}$ respectively (Renganathan et al., 2017). So there is a greater scope for exploiting its nutritional potential of the crop to fight against hidden hunger in developing countries. However systematic analysis of genotypes for their breeding value remains to be undertaken and the genetic studies leading to the understanding of the nature of gene action are also not attempted in this crop. Therefore, there is a need to collect more precise information on the inheritance pattern of grain yield, yield components and micronutrient traits their nature of association with each other in segregating populations in order to formulate dependable selection criteria.

\section{Materials and Methods}

The present study was conducted during 201617 at the experimental plot of Department of Plant Breeding and Genetics, Agricultural College \& Research Institute, Madurai, Tamil Nadu, India. The experimental material included are ACM 331, ACM 333, ACM 335 and MA 10 that utilized for hybridization which is based on variation in iron and zinc content (ACM 331 low in Fe and $\mathrm{Zn}$, where ACM 333 and MA 10 are rich in both), and $F_{2}$ segregating population of ACM $331 \times$ ACM 333, ACM 331 x ACM 335 and ACM 331x MA 10 . The $F_{2}$ segregating population were raised in 40 rows consists of 500 plants with the spacing of $30 \times 10 \mathrm{~cm}$. Individual plant selection was made in each of the three $F_{2}$ population and forwarded to $F_{3}$ with the objective of studying the breeding potential of each cross, parent progeny regression and isolation of some superior lines for yield along with micronutrient traits. Observations were recorded for the following parameters: days to maturity, number of racemes, single ear head weight, number of tillers, thousand grain weight, iron and zinc content, fodder and grain yield. The estimation of micronutrient content was done through Atomic Absorption Spectrophotometer. Selected plants in each $\mathrm{F}_{2}$ cross was raised as family wise. A total of top ten individual plants from each crosses were raised as family. Two rows per family in each cross were maintained and observations were recorded in a random of five plants in each family of the cross. Observed data of $\mathrm{F}_{3}$ generation were subjected to statistical analysis. The simple descriptive statistics such as mean, standardized range, variance and PCV were used as criteria to assess the breeding potential of the selected crosses. The parent progeny regression analysis was carried out by regressing the mean values of a character in the progeny $\left(\mathrm{F}_{3}\right)$ upon the value of a character in the parents $\left(F_{2}\right)$. The regression coefficient ' $b$ ' was calculated by using the formula suggested by Lush (1940).

\section{Results and Discussion}

Development and identification of superior lines derived from heterotic crosses appear to be a better strategy for genetic enhancement of barnyard millet for the traits considered in the present study. The data on different characters along with the estimates on variability indicating genotypic coefficient of variation (GCV), phenotypic coefficient of variation $(\mathrm{PCV})$ and heritability are presented (Table 1). High PCV and GCVs were noticed for number of tillers, iron content, zinc content and fodder 
yield in all the three cross (ACM $331 \times$ ACM 333, ACM 331 x ACM 335, ACM 331 x MA 10). Values of phenotypic and genotypic variance were high for these characters and are indicative of stable nature of these characters. These were concomitant with Govindraj et al., (2011) in pearl millet for grain iron concentration, Akinwale et al., (2011) and Ravindra Babu et al., (2012) in rice for grain yield per plant, Bisne et al., (2009), Brar et al., (2011), Ashok et al., (2013) and Soman et al., (2014) for number of productive tillers per plant, iron content and grain yield. Moderate PCV was obtained for number of raceme, single ear head weight and grain yield for all the same crosses. Low PCV and GCV were observed for days to maturity and thousand grain weight in all the crosses. This suggests that the genetic improvement through selection for these traits may not be always effective. Similar results were obtained in rice by Arpita et al., (2014), Khare et al., (2014), Soman et al., (2014), Bekele et al., (2013) and Laxuman et al., (2010) for days to maturity. According to Ansari et al., (2004), high heritability percentage reflects the large heritable variance which may offer the possibility of improvement through selection. High heritability reported for all the studied traits in three crosses suggesting that environmental factors did not affect greatly the phenotypic performance of these traits.

Crop improvement for grain yield has been achieved through effective use of $F_{2}$ and $F_{3}$ segregating population fixing desirable character combinations (Anilkumar et al., 2011). The parent progeny correlation and regression between two generations shows lesser susceptible to environmental effect and is very useful for selection in segregating population for the development of new improved genotypes (Suwarto et al., 2015). The selection of the plants is effective only when the performance of progeny is more dependable on the performance of the parent.
Lush (1940) suggested that selection of best genotypes based on its genetic potentiality can be ascertained by regression of the progeny mean over the value of corresponding parent. All the characters in this study showed strong correlation and regression between F2 and F3 generation in all crosses (Table 2). It indicated that all characters are having high heritability and this is consistent with earlier report of Vogel et al., (1980) in Indian grasses. Banumathy et al., (2017) and Kahani and Hittalmani (2016) in rice reported positive significant regression and correlation coefficient between all morphological traits of $\mathrm{F}_{2}-\mathrm{F}_{3}$ generation. The positive significant regression and correlation coefficient estimate for all character in F3-F4 generation was also reported by Kavithamani et al., (2013). Based on parent progeny analysis, it can be concluded that selection will be effective in the test materials used in this study as revealed by the significant substantial variations among the genotypes for all the characters observed. Therefore, the $\mathrm{F}_{2}$ of ACM $331 \times$ ACM 333, ACM $331 \times$ ACM 335 and ACM $331 \times$ MA 10 material could be used for the development of potential breeding materials like RILs for grain yield and micronutrient traits and to select for high yielding with maximum micronutrient genotypes along with the feature of female that exhibits stay green type under drought condition.

All crosses showed less coefficient of variation compared to $\mathrm{F}_{2}$ and it indicated the settling down nature of homozygosity in F3 generation. The mean, standard error and coefficient of variation for observed traits of selected F3 families of three crosses were depicted in Table $3 \mathrm{a}, 3 \mathrm{~b}$ and $3 \mathrm{c}$. The cross ACM 331 x ACM 335 showed high Fe and Zn content coupled with moderate yield whereas ACM 331 x MA 10 cross recorded high yield with moderate $\mathrm{Fe}$ and $\mathrm{Zn}$. High $\mathrm{Fe}$ and $\mathrm{Zn}$ content along with low yield was observed in all crosses of families. 
Table.1 Estimates of variability parameters in $\mathrm{F}_{2}$ generation for three crosses

\begin{tabular}{|c|c|c|c|c|c|c|c|c|}
\hline Characters & Cross & Mean & SE & Minimum & Maximum & PCV $(\%)$ & GCV $(\%)$ & $\begin{array}{c}\text { Heritability } \\
\text { (BS) }(\%)\end{array}$ \\
\hline \multirow[t]{3}{*}{ Days to maturity } & ACM 331 x ACM 335 & 99.16 & 0.04 & 90.00 & 106.00 & 4.14 & 4.03 & 94.73 \\
\hline & ACM 331 x ACM 333 & 99.30 & 0.04 & 92.00 & 108 & 4.04 & 3.93 & 94.39 \\
\hline & ACM 331 x MA 10 & 99.89 & 0.04 & 93.00 & 108.00 & 4.07 & 3.94 & 94.04 \\
\hline \multirow[t]{3}{*}{ Number of raceme } & ACM 331 x ACM 335 & 50.77 & 0.13 & 40.00 & 67.00 & 13.42 & 12.75 & 90.26 \\
\hline & ACM 331 x ACM 333 & 54.84 & 0.15 & 41.00 & 73.00 & 14.55 & 14.29 & 96.61 \\
\hline & ACM 331 x MA 10 & 57.22 & 0.12 & 42.00 & 75.00 & 11.88 & 11.31 & 90.62 \\
\hline \multirow{3}{*}{ Single ear head weight (g) } & ACM 331 x ACM 335 & 14.40 & 0.19 & 10.20 & 20.60 & 19.57 & 19.01 & 94.42 \\
\hline & ACM 331 x ACM 333 & 16.86 & 0.20 & 10.73 & 22.60 & 20.20 & 19.17 & 89.95 \\
\hline & ACM 331 x MA 10 & 18.37 & 0.20 & 11.60 & 24.60 & 20.21 & 19.18 & 90.07 \\
\hline \multirow[t]{3}{*}{ Number of tillers } & ACM $331 \times$ ACM 335 & 8.25 & 0.27 & 4.00 & 12.00 & 26.67 & 24.90 & 87.19 \\
\hline & ACM 331 x ACM 333 & 7.24 & 0.30 & 3.00 & 12.00 & 29.46 & 26.88 & 83.29 \\
\hline & ACM 331 x MA 10 & 7.89 & 0.29 & 4.00 & 13.00 & 29.01 & 26.20 & 81.55 \\
\hline \multirow[t]{3}{*}{ Thousand grain weight (g) } & ACM 331 x ACM 335 & 3.20 & 0.14 & 2.80 & 3.63 & 4.74 & 4.12 & 94.75 \\
\hline & ACM 331 x ACM 333 & 3.30 & 0.11 & 2.9 & 3.8 & 6.00 & 5.96 & 90.00 \\
\hline & ACM $331 \times$ MA 10 & 3.20 & 0.12 & 2.80 & 3.80 & 6.0 & 5.85 & 97.43 \\
\hline \multirow[t]{3}{*}{ Iron (mg/100 g) } & ACM 331 x ACM 335 & 13.26 & 0.29 & 7.00 & 18.25 & 28.64 & 28.32 & 97.82 \\
\hline & ACM 331 x ACM 333 & 11.53 & 0.21 & 7.42 & 15.62 & 20.49 & 20.19 & 97.08 \\
\hline & ACM 331 x MA 10 & 11.4 & 0.28 & 6.70 & 16.13 & 25.93 & 25.77 & 98.78 \\
\hline \multirow[t]{3}{*}{ Zinc (mg/100 g) } & ACM 331 x ACM 335 & 5.60 & 0.28 & 2.71 & 7.61 & 27.54 & 26.86 & 95.13 \\
\hline & ACM 331 x ACM 333 & 4.54 & 0.28 & 2.81 & 6.14 & 27.54 & 26.86 & 95.13 \\
\hline & ACM 331 x MA 10 & 4.6 & 0.27 & 2.53 & 7.16 & 24.56 & 23.55 & 91.94 \\
\hline \multirow[t]{3}{*}{ Fodder yield (g) } & ACM 331 x ACM 335 & 54.5 & 0.27 & 34.56 & 103.2 & 30.13 & 28.76 & 76.53 \\
\hline & ACM 331 x ACM 333 & 76.1 & 0.26 & 60.4 & 104.3 & 26.34 & 25.82 & 76.53 \\
\hline & ACM 331 x MA 10 & 70.0 & 0.29 & 40.3 & 103.5 & 29.74 & 28.20 & 81.26 \\
\hline \multirow[t]{3}{*}{ Grain yield (g) } & ACM 331 x ACM 335 & 32.01 & 0.14 & 23.05 & 40.41 & 14.03 & 11.11 & 62.65 \\
\hline & ACM 331 x ACM 333 & 35.97 & 0.21 & 24.11 & 50.16 & 20.39 & 16.88 & 68.51 \\
\hline & ACM 331 x MA 10 & 38.71 & 0.20 & 26.35 & 56.41 & 20.03 & 19.18 & 91.17 \\
\hline
\end{tabular}


Table.2 Parent offspring correlation and regression for different characters in selected three $\mathrm{F}_{2}$ and $\mathrm{F}_{3}$ population

\begin{tabular}{|c|c|c|c|c|c|c|}
\hline \multirow[t]{2}{*}{ Parameters } & \multicolumn{3}{|c|}{ Correlation coefficient } & \multicolumn{3}{|c|}{ Regression coefficient } \\
\hline & $\begin{array}{l}\text { ACM331x } \\
\text { ACM335 }\end{array}$ & $\begin{array}{l}\text { ACM } \\
\text { 331xACM } 333\end{array}$ & $\begin{array}{l}\text { ACM331x } \\
\text { MA10 }\end{array}$ & $\begin{array}{l}\text { ACM331x } \\
\text { ACM335 }\end{array}$ & $\begin{array}{l}\text { ACM } \\
\text { 331xACM } 333\end{array}$ & $\begin{array}{l}\text { ACM331x } \\
\text { MA10 }\end{array}$ \\
\hline Generation & $\mathbf{F}_{2}-\mathbf{F}_{3}$ & $\mathbf{F}_{2}-\mathbf{F}_{3}$ & $F_{2}-F_{3}$ & $\mathbf{F}_{2}-\mathbf{F}_{3}$ & $\mathbf{F}_{2}-\mathbf{F}_{3}$ & $\mathbf{F}_{2}-\mathbf{F}_{3}$ \\
\hline Days to maturity & $0.77^{* *}$ & $0.85^{* *}$ & $0.79^{* *}$ & $0.63^{* *}$ & $0.88^{* * *}$ & $0.61^{* *}$ \\
\hline Number of raceme & $0.80^{* *}$ & $0.85^{* *}$ & $0.91^{* *}$ & $0.58^{* *}$ & $0.74^{* *}$ & $0.66^{* *}$ \\
\hline Single ear head weight (g) & $0.79^{* *}$ & $0.93^{* *}$ & $0.87^{* *}$ & $0.77^{* *}$ & $0.89^{* *}$ & $0.52^{* *}$ \\
\hline Number of tillers & $0.66^{* *}$ & $0.88^{* *}$ & $0.97^{* *}$ & $0.56^{* *}$ & $0.92^{* *}$ & $0.72^{* *}$ \\
\hline $\begin{array}{l}\text { Thousand } \\
\text { Weight(g) }\end{array}$ & $0.58^{* *}$ & $0.71^{* *}$ & $0.65^{* *}$ & $0.91^{* *}$ & $0.88^{* *}$ & $0.75^{* *}$ \\
\hline Iron $(\mathrm{mg} / 100 \mathrm{~g})$ & $0.74^{* * *}$ & $0.66^{* *}$ & $0.67^{* *}$ & $0.78^{* *}$ & $0.48^{* *}$ & $0.46^{* *}$ \\
\hline Zinc (mg/100 g) & $0.96^{* *}$ & $0.73^{* *}$ & $0.71^{* *}$ & $0.77^{* *}$ & $0.68^{* *}$ & $0.52^{* *}$ \\
\hline Fodder Yield (g) & $0.58^{* *}$ & $0.92^{* *}$ & $0.91^{* *}$ & $0.75^{* *}$ & $0.80^{* *}$ & $0.59^{* *}$ \\
\hline Grain yield (g) & $0.94^{* *}$ & $0.90^{* *}$ & $0.94^{* *}$ & $0.52^{* *}$ & $0.50^{* *}$ & $0.61^{* *}$ \\
\hline
\end{tabular}

Table.4 Mean performance of superior families among three crosses

\begin{tabular}{|c|c|c|c|c|c|c|c|c|c|}
\hline Crosses & DM & NR & SEW & NT & TW & Fe & Zn & FY & GY \\
\hline ACM 331 x A CM333 & 4 \& 10 & 4 & 4 & 4 & 4 & 4 & 4 & 5 & 4 \\
& $(96.35)$ & $(56.30)$ & $(18.60)$ & $(9.05)$ & $(3.50)$ & $(13.05)$ & $(5.42)$ & $(91.05)$ & $(46.50)$ \\
\hline \% over control & -7.36 & -2.93 & 9.09 & 81 & 2.94 & 16.52 & 72.06 & 74.76 & 17.72 \\
\hline A CM 331 x A CM335 & 4 & 1 & 9 & 1 & 5 & 5 & 5 & 1 & 6 \\
& $(94.20)$ & $(51.45)$ & $(16.90)$ & $(8.10)$ & $(3.75)$ & $(17.05)$ & $(6.87)$ & $(95.50)$ & $(39.75)$ \\
\hline \% over control & -9.42 & -11.29 & -0.88 & 62 & 10.29 & 52.23 & 118.09 & 83.30 & 0.63 \\
\hline ACM 331 x MA10 & 3 & 3 & 3 & 3 & 3 & 9 & 3 & 4 & 3 \\
& $(95.50)$ & $(60.15)$ & $(20.65)$ & $(9.50)$ & $(3.60)$ & $(15.45)$ & $(6.00)$ & $(98.65)$ & $(49.80)$ \\
\hline$\%$ over control & -8.17 & 3.71 & 21.11 & 90 & 5.88 & 37.95 & 90.48 & 89.34 & 26.08 \\
\hline
\end{tabular}

() Parenthesis-mean values; DM Days to maturity; NR Number of raceme; SEW Single ear head weight; NT Number of tillers; Thousand grain weight; Fe Iron; Zn Zinc; FY Fodder yield per plant; GY Grain yield per plant 
Table.3a Mean, standard error and coefficient of variation for ACM 331 x ACM 335 cross of selected families studied in $\mathrm{F}_{3}$ generation

\begin{tabular}{|c|c|c|c|c|c|c|c|c|c|}
\hline \multirow[t]{2}{*}{ Families } & \multicolumn{9}{|c|}{ ACM331 x ACM335 } \\
\hline & DM & NR & SEW & NT & TW & $\mathrm{Fe}$ & $\mathbf{Z n}$ & FY & GY \\
\hline $\begin{array}{l}\mathrm{Co}(\mathrm{Kv}) 2 \\
\text { (control) }\end{array}$ & $\begin{array}{c}104.00 \\
\pm \\
0.08\end{array}$ & $\begin{array}{c}58.00 \\
\pm \\
0.25\end{array}$ & $\begin{array}{c}17.05 \\
\pm \\
0.10\end{array}$ & $\begin{array}{c}5.00 \\
\pm \\
0.25\end{array}$ & $\begin{array}{c}3.40 \\
\pm \\
0.15\end{array}$ & $\begin{array}{c}11.20 \\
\pm \\
0.24\end{array}$ & $\begin{array}{c}3.15 \\
\pm \\
0.30\end{array}$ & $\begin{array}{c}52.10 \\
\pm \\
0.75\end{array}$ & $\begin{array}{c}39.50 \\
\pm \\
0.40\end{array}$ \\
\hline 1 & $\begin{array}{c}98.48 \\
\pm \\
0.48 \\
(4.86)\end{array}$ & $\begin{array}{c}51.45 \\
\pm \\
1.28 \\
(8.41)\end{array}$ & $\begin{array}{c}16.85 \\
\pm \\
0.48 \\
(5.97)\end{array}$ & $\begin{array}{c}8.10 \\
\pm \\
0.74 \\
(11.69)\end{array}$ & $\begin{array}{c}3.58 \\
\pm \\
1.06 \\
(9.94)\end{array}$ & $\begin{array}{c}14.85 \\
\pm \\
0.75 \\
(5.27)\end{array}$ & $\begin{array}{c}6.34 \\
\pm \\
0.57 \\
(9.33)\end{array}$ & $\begin{array}{c}95.50 \\
\pm \\
1.07 \\
(10.47)\end{array}$ & $\begin{array}{c}37.60 \\
\pm \\
1.95 \\
(10.57)\end{array}$ \\
\hline 2 & $\begin{array}{c}100.05 \\
\pm \\
1.06 \\
(5.62)\end{array}$ & $\begin{array}{c}51.40 \\
\pm \\
1.43 \\
(11.05)\end{array}$ & $\begin{array}{c}16.35 \\
\pm \\
1.74 \\
(11.97)\end{array}$ & $\begin{array}{c}7.70 \\
\pm \\
1.56 \\
(7.66)\end{array}$ & $\begin{array}{c}3.50 \\
\pm \\
0.26 \\
(5.56)\end{array}$ & $\begin{array}{c}13.85 \\
\pm \\
0.69 \\
(8.51)\end{array}$ & $\begin{array}{c}6.05 \\
\pm \\
1.17 \\
(12.8)\end{array}$ & $\begin{array}{c}90.45 \\
\pm \\
0.50 \\
(4.42)\end{array}$ & $\begin{array}{c}36.95 \\
\pm \\
0.82 \\
(11.26)\end{array}$ \\
\hline 3 & $\begin{array}{c}95.50 \\
\pm \\
0.91 \\
(4.57)\end{array}$ & $\begin{array}{c}49.65 \\
\pm \\
1.30 \\
(9.11)\end{array}$ & $\begin{array}{c}14.95 \\
\pm \\
1.90 \\
(10.05)\end{array}$ & $\begin{array}{c}6.10 \\
\pm \\
0.73 \\
(8.28)\end{array}$ & $\begin{array}{c}3.24 \\
\pm \\
0.36 \\
(5.10)\end{array}$ & $\begin{array}{c}12.45 \\
\pm \\
1.19 \\
(16.48)\end{array}$ & $\begin{array}{c}4.99 \\
\pm \\
0.52 \\
(6.67)\end{array}$ & $\begin{array}{c}80.50 \\
\pm \\
1.52 \\
(11.66)\end{array}$ & $\begin{array}{c}35.90 \\
\pm \\
0.82 \\
(7.33)\end{array}$ \\
\hline 4 & $\begin{array}{c}94.20 \\
\pm \\
1.03 \\
(4.93)\end{array}$ & $\begin{array}{c}51.05 \\
\pm \\
0.72 \\
(6.45)\end{array}$ & $\begin{array}{c}15.95 \\
\pm \\
1.96 \\
(6.92)\end{array}$ & $\begin{array}{c}6.90 \\
\pm \\
0.69 \\
(10.26)\end{array}$ & $\begin{array}{c}3.45 \\
\pm \\
1.16 \\
(9.36)\end{array}$ & $\begin{array}{c}11.80 \\
\pm \\
0.26 \\
(5.54)\end{array}$ & $\begin{array}{c}4.05 \\
\pm \\
1.62 \\
(10.07)\end{array}$ & $\begin{array}{c}65.95 \\
\pm \\
0.76 \\
(12.26)\end{array}$ & $\begin{array}{c}36.35 \\
\pm \\
1.50 \\
(8.79)\end{array}$ \\
\hline 5 & $\begin{array}{c}95.10 \\
\pm \\
1.69 \\
(4.77)\end{array}$ & $\begin{array}{c}49.35 \\
\pm \\
0.76 \\
(6.93)\end{array}$ & $\begin{array}{c}15.05 \\
\pm \\
0.98 \\
(11.83)\end{array}$ & $\begin{array}{c}7.00 \\
\pm \\
1.42 \\
(9.59)\end{array}$ & $\begin{array}{c}3.75 \\
\pm \\
0.73 \\
(8.95)\end{array}$ & $\begin{array}{c}17.05 \\
\pm \\
0.57 \\
(9.51)\end{array}$ & $\begin{array}{c}6.87 \\
\pm \\
1.56 \\
(9.27)\end{array}$ & $\begin{array}{c}90.05 \\
\pm \\
0.93 \\
(6.57)\end{array}$ & $\begin{array}{c}36.80 \\
\pm \\
1.91 \\
(9.33)\end{array}$ \\
\hline 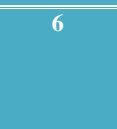 & $\begin{array}{c}96.00 \\
\pm \\
0.88 \\
(5.61)\end{array}$ & $\begin{array}{c}50.70 \\
\pm \\
1.75 \\
(6.71)\end{array}$ & $\begin{array}{c}16.20 \\
\pm \\
1.29 \\
(12.88)\end{array}$ & $\begin{array}{c}7.50 \\
\pm \\
1.21 \\
(7.49)\end{array}$ & $\begin{array}{c}3.54 \\
\pm \\
0.94 \\
(9.26)\end{array}$ & $\begin{array}{c}12.29 \\
\pm \\
1.33 \\
(5.47)\end{array}$ & $\begin{array}{c}5.35 \\
\pm \\
1.13 \\
(9.39)\end{array}$ & $\begin{array}{c}87.90 \\
\pm \\
1.28 \\
(8.56)\end{array}$ & $\begin{array}{c}39.75 \\
\pm \\
1.68 \\
(10.75)\end{array}$ \\
\hline 7 & $\begin{array}{c}97.00 \\
\pm \\
0.96 \\
(6.68)\end{array}$ & $\begin{array}{c}51.25 \\
\pm \\
1.01 \\
(10.15)\end{array}$ & $\begin{array}{c}16.45 \\
\pm \\
0.59 \\
(5.86)\end{array}$ & $\begin{array}{c}7.20 \\
\pm \\
1.32 \\
(11.68)\end{array}$ & $\begin{array}{c}3.51 \\
\pm \\
0.57 \\
(10.49)\end{array}$ & $\begin{array}{c}16.78 \\
\pm \\
1.61 \\
(9.78)\end{array}$ & $\begin{array}{c}6.01 \\
\pm \\
1.36 \\
(6.00)\end{array}$ & $\begin{array}{c}75.40 \\
\pm \\
0.57 \\
(5.36)\end{array}$ & $\begin{array}{c}39.20 \\
\pm \\
0.53 \\
(6.24)\end{array}$ \\
\hline 8 & $\begin{array}{c}101.00 \\
\pm \\
0.41 \\
(7.93)\end{array}$ & $\begin{array}{c}50.86 \\
\pm \\
0.53 \\
(7.63)\end{array}$ & $\begin{array}{c}15.85 \\
\pm \\
0.66 \\
(5.05)\end{array}$ & $\begin{array}{c}6.40 \\
\pm \\
1.04 \\
(6.75)\end{array}$ & $\begin{array}{c}3.25 \\
\pm \\
0.39 \\
(10.17)\end{array}$ & $\begin{array}{c}13.20 \\
\pm \\
1.20 \\
(7.17)\end{array}$ & $\begin{array}{c}4.95 \\
\pm \\
0.69 \\
(5.05)\end{array}$ & $\begin{array}{c}69.85 \\
\pm \\
0.88 \\
(10.83)\end{array}$ & $\begin{array}{c}36.00 \\
\pm \\
0.53 \\
(6.70)\end{array}$ \\
\hline 9 & $\begin{array}{c}99.20 \\
\pm \\
1.40 \\
(4.52)\end{array}$ & $\begin{array}{c}50.95 \\
\pm \\
1.01 \\
(10.15)\end{array}$ & $\begin{array}{c}16.90 \\
\pm \\
0.76 \\
(6.36)\end{array}$ & $\begin{array}{c}7.00 \\
\pm \\
0.48 \\
(8.33)\end{array}$ & $\begin{array}{c}3.45 \\
\pm \\
0.63 \\
(12.20)\end{array}$ & $\begin{array}{c}14.05 \\
\pm \\
1.17 \\
(9.56)\end{array}$ & $\begin{array}{c}5.75 \\
\pm \\
0.81 \\
(11.77)\end{array}$ & $\begin{array}{c}70.50 \\
\pm \\
1.32 \\
(8.97)\end{array}$ & $\begin{array}{c}36.70 \\
\pm \\
1.16 \\
(10.23)\end{array}$ \\
\hline 10 & $\begin{array}{c}98.30 \\
\pm \\
0.36 \\
(4.31)\end{array}$ & $\begin{array}{c}49.05 \\
\pm \\
1.09 \\
(7.63)\end{array}$ & $\begin{array}{c}14.50 \\
\pm \\
1.07 \\
(12.27)\end{array}$ & $\begin{array}{c}6.25 \\
\pm \\
0.36 \\
(8.16)\end{array}$ & $\begin{array}{c}3.10 \\
\pm \\
0.79 \\
(11.98)\end{array}$ & $\begin{array}{c}13.95 \\
\pm \\
1.02 \\
(9.10)\end{array}$ & $\begin{array}{c}5.5 \\
\pm \\
1.44 \\
(5.92\end{array}$ & $\begin{array}{c}65.40 \\
\pm \\
1.29 \\
(10.23)\end{array}$ & $\begin{array}{c}35.95 \\
\pm \\
0.69 \\
(12.27)\end{array}$ \\
\hline
\end{tabular}

() Parenthesis-coefficient of variation; DM Days to maturity; NR Number of raceme; SEW Single ear head weight; NT Number of tillers; Thousand grain weight; Fe Iron; Zn Zinc; FY Fodder yield per plant; GY Grain yield per plant 
Table.3b Mean, standard error and coefficient of variation for ACM 331 x ACM 333 cross of selected families studied in $\mathrm{F}_{3}$ generation

\begin{tabular}{|c|c|c|c|c|c|c|c|c|c|}
\hline \multirow[t]{2}{*}{ Families } & \multicolumn{9}{|c|}{ ACM331 x ACM333 } \\
\hline & DM & NR & SEW & NT & TW & $\mathrm{Fe}$ & $\mathbf{Z n}$ & FY & GY \\
\hline $\begin{array}{l}\text { Co }(\mathbf{K v}) 2 \\
\text { (control) }\end{array}$ & $\begin{array}{c}104.00 \\
\pm \\
0.08\end{array}$ & $\begin{array}{c}58.00 \\
\pm \\
0.25\end{array}$ & $\begin{array}{c}17.05 \\
\pm \\
0.10\end{array}$ & $\begin{array}{c}5.00 \\
\pm \\
0.25\end{array}$ & $\begin{array}{c}3.40 \\
\pm \\
0.15\end{array}$ & $\begin{array}{c}11.20 \\
\pm \\
0.24\end{array}$ & $\begin{array}{c}3.15 \\
\pm \\
0.30\end{array}$ & $\begin{array}{c}52.10 \\
\pm \\
0.75\end{array}$ & $\begin{array}{c}39.50 \\
\pm \\
0.40\end{array}$ \\
\hline 1 & $\begin{array}{c}97.85 \\
\pm \\
0.08 \\
(5.31)\end{array}$ & $\begin{array}{c}53.65 \\
\pm \\
0.24 \\
(8.75)\end{array}$ & $\begin{array}{c}16.80 \\
\pm \\
0.12 \\
(6.82)\end{array}$ & $\begin{array}{c}8.05 \\
\pm \\
0.42 \\
(10.38)\end{array}$ & $\begin{array}{c}3.37 \\
\pm \\
0.24 \\
(7.39)\end{array}$ & $\begin{array}{c}12.00 \\
\pm \\
0.13 \\
(5.27)\end{array}$ & $\begin{array}{c}4.98 \\
\pm \\
0.24 \\
(9.59)\end{array}$ & $\begin{array}{c}87.56 \\
\pm \\
0.26 \\
(9.09)\end{array}$ & $\begin{array}{c}44.26 \\
\pm \\
0.38 \\
(8.55)\end{array}$ \\
\hline 2 & $\begin{array}{c}98.65 \\
\pm \\
0.43 \\
(6.52)\end{array}$ & $\begin{array}{c}54.25 \\
\pm \\
0.35 \\
(8.94)\end{array}$ & $\begin{array}{c}17.65 \\
\pm \\
0.27 \\
(7.23)\end{array}$ & $\begin{array}{c}8.95 \\
\pm \\
0.42 \\
(11.54)\end{array}$ & $\begin{array}{c}3.43 \\
\pm \\
0.16 \\
(5.89)\end{array}$ & $\begin{array}{c}12.65 \\
\pm \\
0.32 \\
(8.82)\end{array}$ & $\begin{array}{c}5.05 \\
\pm \\
0.24 \\
(8.22)\end{array}$ & $\begin{array}{c}86.40 \\
\pm \\
0.16 \\
(6.64)\end{array}$ & $\begin{array}{c}43.68 \\
\pm \\
0.27 \\
(8.81)\end{array}$ \\
\hline 3 & $\begin{array}{c}99.45 \\
\pm \\
0.20 \\
(5.52)\end{array}$ & $\begin{array}{c}55.40 \\
\pm \\
0.39 \\
(8.73)\end{array}$ & $\begin{array}{c}18.25 \\
\pm \\
0.24 \\
(7.80)\end{array}$ & $\begin{array}{c}9.00 \\
\pm \\
0.24 \\
(10.99)\end{array}$ & $\begin{array}{c}3.40 \\
\pm \\
0.14 \\
(6.98)\end{array}$ & $\begin{array}{c}10.50 \\
\pm \\
0.23 \\
(10.69)\end{array}$ & $\begin{array}{c}3.76 \\
\pm \\
0.18 \\
(5.76)\end{array}$ & $\begin{array}{c}88.24 \\
\pm \\
0.31 \\
(10.92)\end{array}$ & $\begin{array}{c}45.30 \\
\pm \\
0.21 \\
(7.40)\end{array}$ \\
\hline 4 & $\begin{array}{c}96.35 \\
\pm \\
0.25 \\
(4.16)\end{array}$ & $\begin{array}{c}56.30 \\
\pm \\
0.16 \\
(11.59)\end{array}$ & $\begin{array}{c}18.60 \\
\pm \\
0.18 \\
(7.81)\end{array}$ & $\begin{array}{c}9.05 \\
\pm \\
0.39 \\
(15.57)\end{array}$ & $\begin{array}{c}3.50 \\
\pm \\
0.27 \\
(10.22)\end{array}$ & $\begin{array}{c}13.05 \\
\pm \\
0.11 \\
(8.14)\end{array}$ & $\begin{array}{c}5.42 \\
\pm \\
0.41 \\
(10.63)\end{array}$ & $\begin{array}{c}90.15 \\
\pm \\
0.34 \\
(12.03)\end{array}$ & $\begin{array}{c}46.50 \\
\pm \\
0.26 \\
(9.67)\end{array}$ \\
\hline 5 & $\begin{array}{c}98.60 \\
\pm \\
0.26 \\
(6.33)\end{array}$ & $\begin{array}{c}52.40 \\
\pm \\
0.16 \\
(12.30)\end{array}$ & $\begin{array}{c}16.85 \\
\pm \\
0.25 \\
(6.97)\end{array}$ & $\begin{array}{c}7.80 \\
\pm \\
0.29 \\
(9.68)\end{array}$ & $\begin{array}{c}3.45 \\
\pm \\
0.23 \\
(7.84)\end{array}$ & $\begin{array}{c}11.90 \\
\pm \\
0.22 \\
(7.04)\end{array}$ & $\begin{array}{c}5.24 \\
\pm \\
0.76 \\
(12.44)\end{array}$ & $\begin{array}{c}91.05 \\
\pm \\
0.24 \\
(10.83)\end{array}$ & $\begin{array}{c}45.25 \\
\pm \\
0.30 \\
(10.17)\end{array}$ \\
\hline 6 & $\begin{array}{c}99.75 \\
\pm \\
0.29 \\
(7.28)\end{array}$ & $\begin{array}{c}53.78 \\
\pm \\
0.38 \\
(9.96)\end{array}$ & $\begin{array}{c}17.45 \\
\pm \\
0.44 \\
(11.94)\end{array}$ & $\begin{array}{c}8.00 \\
\pm \\
0.11 \\
(6.58)\end{array}$ & $\begin{array}{c}3.25 \\
\pm \\
0.20 \\
(8.61)\end{array}$ & $\begin{array}{c}12.43 \\
\pm \\
0.27 \\
(10.73)\end{array}$ & $\begin{array}{c}5.13 \\
\pm \\
0.27 \\
(7.94)\end{array}$ & $\begin{array}{c}85.60 \\
\pm \\
0.39 \\
(10.83)\end{array}$ & $\begin{array}{c}42.90 \\
\pm \\
0.33 \\
(8.58)\end{array}$ \\
\hline 7 & $\begin{array}{c}100.50 \\
\pm \\
0.32 \\
(4.26)\end{array}$ & $\begin{array}{c}54.60 \\
\pm \\
0.23 \\
(9.01)\end{array}$ & $\begin{array}{c}17.90 \\
\pm \\
0.12 \\
(8.40)\end{array}$ & $\begin{array}{c}8.65 \\
\pm \\
0.28 \\
(9.58)\end{array}$ & $\begin{array}{c}3.24 \\
\pm \\
0.26 \\
(8.41)\end{array}$ & $\begin{array}{c}10.85 \\
\pm \\
0.23 \\
(7.35)\end{array}$ & $\begin{array}{c}4.08 \\
\pm \\
0.24 \\
(7.99)\end{array}$ & $\begin{array}{c}84.25 \\
\pm \\
0.12 \\
(9.37)\end{array}$ & $\begin{array}{c}44.75 \\
\pm \\
0.12 \\
(6.20)\end{array}$ \\
\hline 8 & $\begin{array}{c}97.60 \\
\pm \\
0.14 \\
(4.41)\end{array}$ & $\begin{array}{c}53.80 \\
\pm \\
0.21 \\
(8.28)\end{array}$ & $\begin{array}{c}16.95 \\
\pm \\
0.33 \\
(7.44)\end{array}$ & $\begin{array}{c}7.90 \\
\pm \\
0.18 \\
(5.79)\end{array}$ & $\begin{array}{c}3.42 \\
\pm \\
0.25 \\
(10.65)\end{array}$ & $\begin{array}{c}11.90 \\
\pm \\
0.22 \\
(8.57)\end{array}$ & $\begin{array}{c}4.90 \\
\pm \\
0.14 \\
(1.69)\end{array}$ & $\begin{array}{c}80.78 \\
\pm \\
0.24 \\
(9.33)\end{array}$ & $\begin{array}{c}43.90 \\
\pm \\
0.15 \\
(7.44)\end{array}$ \\
\hline 9 & $\begin{array}{c}98.20 \\
\pm \\
0.25 \\
(4.21)\end{array}$ & $\begin{array}{c}54.35 \\
\pm \\
0.23 \\
(9.01)\end{array}$ & $\begin{array}{c}17.85 \\
\pm \\
0.26 \\
(9.48)\end{array}$ & $\begin{array}{c}8.20 \\
\pm \\
0.36 \\
(8.24)\end{array}$ & $\begin{array}{c}3.45 \\
\pm \\
0.33 \\
(11.23)\end{array}$ & $\begin{array}{c}12.35 \\
\pm \\
0.27 \\
(5.09)\end{array}$ & $\begin{array}{c}4.80 \\
\pm \\
0.30 \\
(10.78)\end{array}$ & $\begin{array}{c}85.40 \\
\pm \\
0.25 \\
(9.27)\end{array}$ & $\begin{array}{c}42.65 \\
\pm \\
0.28 \\
(9.29)\end{array}$ \\
\hline$\overline{10}$ & $\begin{array}{c}96.35 \\
\pm \\
0.12 \\
(7.81)\end{array}$ & $\begin{array}{c}55.00 \\
\pm \\
0.21 \\
(8.28)\end{array}$ & $\begin{array}{c}18.00 \\
\pm \\
0.29 \\
(8.59)\end{array}$ & $\begin{array}{c}8.50 \\
\pm \\
0.12 \\
(8.86)\end{array}$ & $\begin{array}{c}3.48 \\
\pm \\
0.35 \\
(11.51)\end{array}$ & $\begin{array}{c}12.28 \\
\pm \\
0.13 \\
(7.95)\end{array}$ & $\begin{array}{c}5.15 \\
\pm \\
0.37 \\
(9.59)\end{array}$ & $\begin{array}{c}89.35 \\
\pm \\
0.23 \\
(8.99)\end{array}$ & $\begin{array}{c}44.65 \\
\pm \\
0.29 \\
(10.32)\end{array}$ \\
\hline
\end{tabular}

() Parenthesis-coefficient of variation; DM Days to maturity; NR Number of raceme; SEW Single ear head weight; NT Number of tillers; Thousand grain weight; Fe Iron; Zn Zinc; FY Fodder yield per plant; GY Grain yield per plant 
Table.3c Mean, standard error and coefficient of variation for ACM331 x MA10 cross of selected families studied in $\mathrm{F}_{3}$ generation

\begin{tabular}{|c|c|c|c|c|c|c|c|c|c|}
\hline \multirow{2}{*}{ Families } & \multicolumn{9}{|c|}{ ACM331 x MA10 } \\
\hline & DM & NR & SEW & NT & TW & $\mathrm{Fe}$ & Zn & FY & GY \\
\hline $\begin{array}{l}\text { Co (Kv) } 2 \\
\text { (control) }\end{array}$ & $\begin{array}{c}104.00 \\
\pm \\
0.08\end{array}$ & $\begin{array}{c}58.00 \\
\pm \\
0.25\end{array}$ & $\begin{array}{c}17.05 \\
\pm \\
0.10\end{array}$ & $\begin{array}{c}5.00 \\
\pm \\
0.25\end{array}$ & $\begin{array}{c}3.40 \\
\pm \\
0.15\end{array}$ & $\begin{array}{c}11.20 \\
\pm \\
0.24\end{array}$ & $\begin{array}{c}3.15 \\
\pm \\
0.30\end{array}$ & $\begin{array}{c}52.10 \\
\pm \\
0.75\end{array}$ & $\begin{array}{c}39.50 \\
\pm \\
0.40\end{array}$ \\
\hline 1 & $\begin{array}{c}98.50 \\
\pm \\
0.07 \\
(4.41)\end{array}$ & $\begin{array}{c}58.25 \\
\pm \\
0.08 \\
(10.25)\end{array}$ & $\begin{array}{c}19.65 \\
\pm \\
0.08 \\
(6.54)\end{array}$ & $\begin{array}{c}8.90 \\
\pm \\
0.15 \\
(9.77)\end{array}$ & $\begin{array}{c}3.25 \\
\pm \\
0.10 \\
(11.87)\end{array}$ & $\begin{array}{c}11.00 \\
\pm \\
0.08 \\
(12.33)\end{array}$ & $\begin{array}{c}4.10 \\
\pm \\
0.09 \\
(7.09)\end{array}$ & $\begin{array}{c}83.95 \\
\pm \\
0.13 \\
(8.69)\end{array}$ & $\begin{array}{c}45.85 \\
\pm \\
0.1 \\
(10.88)\end{array}$ \\
\hline 2 & $\begin{array}{c}97.00 \\
\pm \\
0.10 \\
(6.54)\end{array}$ & $\begin{array}{c}57.45 \\
\pm \\
0.15 \\
(10.53)\end{array}$ & $\begin{array}{c}19.20 \\
\pm \\
0.12 \\
(11.99)\end{array}$ & $\begin{array}{c}8.50 \\
\pm \\
0.11 \\
(10.39)\end{array}$ & $\begin{array}{c}3.38 \\
\pm \\
0.07 \\
(14.23)\end{array}$ & $\begin{array}{c}12.80 \\
\pm \\
0.11 \\
(7.04)\end{array}$ & $\begin{array}{c}4.25 \\
\pm \\
0.14 \\
(10.82)\end{array}$ & $\begin{array}{c}85.30 \\
\pm \\
0.08 \\
(5.79)\end{array}$ & $\begin{array}{c}44.63 \\
\pm \\
0.13 \\
(10.27)\end{array}$ \\
\hline 3 & $\begin{array}{c}95.50 \\
\pm \\
0.04 \\
(6.96)\end{array}$ & $\begin{array}{c}60.15 \\
\pm \\
0.10 \\
(9.44)\end{array}$ & $\begin{array}{c}20.65 \\
\pm \\
0.15 \\
(12.23)\end{array}$ & $\begin{array}{c}9.50 \\
\pm \\
0.11 \\
(9.36)\end{array}$ & $\begin{array}{c}3.60 \\
\pm \\
0.07 \\
(10.28)\end{array}$ & $\begin{array}{c}15.15 \\
\pm \\
0.07 \\
(11.15)\end{array}$ & $\begin{array}{c}6.00 \\
\pm \\
0.06 \\
(6.32)\end{array}$ & $\begin{array}{c}92.35 \\
\pm \\
0.11 \\
(10.20)\end{array}$ & $\begin{array}{c}49.80 \\
\pm \\
0.10 \\
(9.35)\end{array}$ \\
\hline 4 & $\begin{array}{c}98.00 \\
\pm \\
0.07 \\
(9.97)\end{array}$ & $\begin{array}{c}58.20 \\
\pm \\
0.08 \\
(11.49)\end{array}$ & $\begin{array}{c}19.00 \\
\pm \\
0.10 \\
(12.79)\end{array}$ & $\begin{array}{c}9.00 \\
\pm \\
0.13 \\
(8.74)\end{array}$ & $\begin{array}{c}3.54 \\
\pm \\
0.12 \\
(9.93)\end{array}$ & $\begin{array}{c}13.05 \\
\pm \\
0.06 \\
(9.37)\end{array}$ & $\begin{array}{c}5.85 \\
\pm \\
0.11 \\
(10.62)\end{array}$ & $\begin{array}{c}98.65 \\
\pm \\
0.08 \\
(6.96)\end{array}$ & $\begin{array}{c}48.20 \\
\pm \\
0.12 \\
(11.29)\end{array}$ \\
\hline 5 & $\begin{array}{c}99.00 \\
\pm \\
0.05 \\
(9.89)\end{array}$ & $\begin{array}{c}57.60 \\
\pm \\
0.08 \\
(12.76)\end{array}$ & $\begin{array}{c}18.95 \\
\pm \\
0.13 \\
(8.69)\end{array}$ & $\begin{array}{c}8.40 \\
\pm \\
0.09 \\
(10.83)\end{array}$ & $\begin{array}{c}3.41 \\
\pm \\
0.05 \\
(8.05)\end{array}$ & $\begin{array}{c}12.85 \\
\pm \\
0.10 \\
(8.06)\end{array}$ & $\begin{array}{c}4.95 \\
\pm \\
0.12 \\
(10.42)\end{array}$ & $\begin{array}{c}90.45 \\
\pm \\
0.10 \\
(6.98)\end{array}$ & $\begin{array}{c}44.89 \\
\pm \\
0.10 \\
(10.49)\end{array}$ \\
\hline 6 & $\begin{array}{c}100.00 \\
\pm \\
0.06 \\
(5.94)\end{array}$ & $\begin{array}{c}58.30 \\
\pm \\
0.05 \\
(10.49)\end{array}$ & $\begin{array}{c}19.10 \\
\pm \\
0.11 \\
(10.98)\end{array}$ & $\begin{array}{c}8.75 \\
\pm \\
0.10 \\
(7.14)\end{array}$ & $\begin{array}{c}3.48 \\
\pm \\
0.07 \\
(9.69)\end{array}$ & $\begin{array}{c}11.01 \\
\pm \\
0.13 \\
(9.12)\end{array}$ & $\begin{array}{c}4.30 \\
\pm \\
0.16 \\
(9.03)\end{array}$ & $\begin{array}{c}96.30 \\
\pm \\
0.07 \\
(9.71)\end{array}$ & $\begin{array}{c}46.10 \\
\pm \\
0.11 \\
(12.20)\end{array}$ \\
\hline 7 & $\begin{array}{c}99.00 \\
\pm \\
0.10 \\
(7.62)\end{array}$ & $\begin{array}{c}56.90 \\
\pm \\
0.10 \\
(9.45)\end{array}$ & $\begin{array}{c}18.34 \\
\pm \\
0.09 \\
(12.35)\end{array}$ & $\begin{array}{c}8.20 \\
\pm \\
0.12 \\
(13.20)\end{array}$ & $\begin{array}{c}3.25 \\
\pm \\
0.09 \\
(6.62)\end{array}$ & $\begin{array}{c}12.90 \\
\pm \\
0.09 \\
(9.34)\end{array}$ & $\begin{array}{c}4.90 \\
\pm \\
0.13 \\
(10.24)\end{array}$ & $\begin{array}{c}85.30 \\
\pm \\
0.10 \\
(14.61)\end{array}$ & $\begin{array}{c}45.85 \\
\pm \\
0.08 \\
(5.45)\end{array}$ \\
\hline 8 & $\begin{array}{c}96.50 \\
\pm \\
0.09 \\
(5.98)\end{array}$ & $\begin{array}{c}59.00 \\
\pm \\
0.10 \\
(6.80)\end{array}$ & $\begin{array}{c}19.85 \\
\pm \\
0.07 \\
(11.65)\end{array}$ & $\begin{array}{c}8.95 \\
\pm \\
0.08 \\
(9.03)\end{array}$ & $\begin{array}{c}3.39 \\
\pm \\
0.08 \\
(7.20)\end{array}$ & $\begin{array}{c}12.78 \\
\pm \\
0.11 \\
(8.67)\end{array}$ & $\begin{array}{c}3.98 \\
\pm \\
0.08 \\
(14.95)\end{array}$ & $\begin{array}{c}92.10 \\
\pm \\
0.11 \\
(7.97)\end{array}$ & $\begin{array}{c}47.00 \\
\pm \\
0.08 \\
(10.67)\end{array}$ \\
\hline 9 & $\begin{array}{c}97.50 \\
\pm \\
0.07 \\
(8.41)\end{array}$ & $\begin{array}{c}53.00 \\
\pm \\
0.10 \\
(9.45)\end{array}$ & $\begin{array}{c}17.90 \\
\pm \\
0.09 \\
(9.80)\end{array}$ & $\begin{array}{c}7.90 \\
\pm \\
0.05 \\
(8.41)\end{array}$ & $\begin{array}{c}3.30 \\
\pm \\
0.12 \\
(11.76)\end{array}$ & $\begin{array}{c}15.45 \\
\pm \\
0.09 \\
(10.22)\end{array}$ & $\begin{array}{c}5.87 \\
\pm \\
0.14 \\
(7.08)\end{array}$ & $\begin{array}{c}82.15 \\
\pm \\
0.11 \\
(10.83)\end{array}$ & $\begin{array}{c}43.60 \\
\pm \\
0.06 \\
(9.93)\end{array}$ \\
\hline$\overline{10}$ & $\begin{array}{c}103.00 \\
\pm \\
4.42 \\
(6.29)\end{array}$ & $\begin{array}{c}54.00 \\
\pm \\
0.10 \\
(12.91)\end{array}$ & $\begin{array}{c}18.20 \\
\pm \\
0.12 \\
(8.92)\end{array}$ & $\begin{array}{c}8.60 \\
\pm \\
0.07 \\
(9.20)\end{array}$ & $\begin{array}{c}3.40 \\
\pm \\
0.15 \\
(9.88)\end{array}$ & $\begin{array}{c}12.55 \\
\pm \\
0.11 \\
(8.76)\end{array}$ & $\begin{array}{c}4.65 \\
\pm \\
0.09 \\
(10.16)\end{array}$ & $\begin{array}{c}88.60 \\
\pm \\
0.12 \\
(10.53)\end{array}$ & $\begin{array}{c}45.90 \\
\pm \\
0.15 \\
(7.04)\end{array}$ \\
\hline
\end{tabular}

() Parenthesis-coefficient of variation; DM Days to maturity; NR Number of raceme; SEW Single ear head weight; NT Number of tillers; Thousand grain weight; Fe Iron; Zn Zinc; FY Fodder yield per plant; GY Grain yield per plant 
This showed that grain yield and micronutrient traits are having non-significant association. The non-significant correlation of grain $\mathrm{Fe}$ concentration with grain yield per plant was reported by Nagesh et al., (2012) in rice and Govindaraj et al., (2013) in maize supporting the present study. It may be used for hybridization programme for improvement of $\mathrm{Fe}$ and $\mathrm{Zn}$ in high yielding varieties. In the F3 generation, the identified superior families in all the three crosses revealed that yield and yield component characters progressively increased when compared to control [Co (Kv) 2] except for days to maturity and number of racemes (ACM 331 x ACM335, ACM $331 \quad x$ ACM333) and single ear head weight (ACM $331 \times$ ACM335). Among the families of ACM $331 \times$ MA10 cross, third, fourth and ninth exhibited superior performance than the control for most of the yield attributing and micronutrient traits and low co efficient variation. These selected families are recommended for promoting to advanced generation for varietal development program with respect to high yield coupled with high iron and zinc content. The superior performances of families are presented in Table 3c.

In the present investigation, in order to isolate high yielding barnyard millet genotypes with maximum micronutrient content simultaneously, these three crosses were analyzed for variability in $\mathrm{F}_{2}$ population. The high PCV and GCV values and high heritability were noticed for number of tillers, iron and zinc content in all the crosses, indicated the predominance of additive gene effects in their expression and would respond to selection effectively as they are least influenced by environment. All these crosses showed less coefficient of variation compared to F2, suggested that the settling down nature of homozygosity in $\mathrm{F}_{3}$ generation. Superior families from ACM 331 x MA 10 that exhibited higher mean values than the parent and local check [CO (Kv) 2] for most yield attributing and micronutrient traits were selected for promoting to advanced generation.

\section{Acknowledgment}

Authors sincerely acknowledge the University Grant Commission for providing financial assistance to carry out the research successfully.

\section{References}

Abe, T., M. Fukami and M. Ogasawara. 2011. Effect of hymexazole (3-hydroxy-5mehtylisoxazole) on cadmium stress and accumulation in Japanese millet (Echinochloa frumentacea Link). J. Pestic. Sci., 36 (1): 48-52.

Akinwale. M.G., Gregorio. G., Nwilene. F., Akinyele. B.O., Ogunbayo. S.A. and Odiyi. A.C. 2011. Heritability and correlation coefficient analysis for yield and its components in rice (Oryza sativa L). Afr. J. Plant Sci., 5: 207-212.

Anilkumar, C. Vanniarajan and J. Ramalingam. 2011. Parent Progeny regression analysis in $\mathrm{F}_{2}$ and $\mathrm{F}_{3}$ generations of rice. Electron. J. Plant. Breed, 2(4): 520-522.

Ansari, K.A., B.A. Ansari and A. Khund. 2004. Extent of heterosis and heritability in rice for yield and physiological characters in rice hybrids. Oryza, 44 (1): 7- 13.

Arpita, S., D. K. Mishra, G. K. Koutu and S. K. Singh. 2014. Heritability and genetic advance estimation from parental lines of hybrid rice. Int. J. Sci. Res., 3(7):1113.

Ashok. K.T., Singh. S. K., Amita Sharma and Bhati. P.K. 2013. Appraisal of genetic variability for yield and its component 
characters in rice (Oryza sativa L.). Biolife, 1(3):84-89.

Bandyopadhyay, B.B. 2009. Yield variation and associated changes in relationship of component characters of a cold sensitive finger millet genotype in subsequent generation. Indian J. Agric. Res., 43(1): 32-36.

Banumathy, S., A. Sheeba, K. Veni and R. Agila. 2017. Parent progeny regression analysis in the segregating generations of rice (Oryza sativa L.) Green Farming, 8 (5): 1044-1047

Bisne. R., Sarawgi. A.K. and S.B. Verulkar. 2009. Study of heritability genetic advance and variability for yield contributing characters in rice. Bangladesh. J. Agril. Res., 34: 175-179.

Brar. B., Jain. S., Singh. R. and Jain. R.K. 2011. Genetic diversity for iron and zinc contents in a collection of 220 rice (Oryza sativa. L) genotypes. Indian $\mathrm{J}$. Genet., 71(1):67-73.

Govindaraj, M., Rai, K.N., Shanmugasundaram, P., Dwivedi, S.L., Sahrawat, K.L., Muthaiah, A.R and Rao, A.S. 2013. Combining ability and heterosis for grain iron and zinc densities in pearl millet. Crop Sci. 53: 507-517.

Govindraj M., Selvi. B., Rajarathinam S. and Sumathi. P., 2011. Genetic variability and heritability of grain yield components and grain mineral concentration in India's pearl millet [Pennisetum glaucum. (L) R. Br.] accessions. Afr. J. Food, Agr. Nutr. Develop, 11(3): 4758-4771.

Gupta, A., V. Mahajan and H. S. Gupta. 2010. Genetic resources and varietal improvement of small millets for Indian Himalaya. In: L. M. Tewari, Y. P. S. Pangtey and G. Tewari (Eds), Biodiversity Potentials of the Himalaya, 305-316. Gyanodaya Prakashan, Nainital, India.
Kahani, F and S. Hittalmani. 2016. Identification of $F_{2}$ and $F_{3}$ segregants of fifteen rice rosses suitable for cultivation under aerobic situation. SABRAO Journal of Breed. and Genetic., 48 (2): 219-229.

Kavithamani D, S. Robin, S. Manonmani and K. Mohanasundaram. 2013. Character association and parent progeny regression studies for yield in the segregating generations of TGMS rice lines. Oryza. 50 (1): 45-51.

Khare R., A.K. Singh, S. Eram and P.K.Singh. 2014. Genetic variability, association and diversity analysis in upland rice [Oryza sativai (L.)]. SAARC J. Agri., 13(1): 99-108

Laxuman P, M., H. Salimath, E. Shashidhar, H. D. Mohankumar, S. S.Patil H. M. Vamadeviha and B. S. Janagoudar, 2010. Analysis of genetic variability in interspecific backcross inbred lines in rice (Oryza sativa L.). The J. of Res. ANGRAU, 41(1):132-134.

Lush, J. L. 1940. Intra - sire correlation and regression of offspring on dams as a method of estimating heritability of characters. In: Proc. of "American Society of Animal Production" 33: 293 -301 .

Nagesh, V. Ravindrababu, G. Usharani and T. Dayakar Reddy. 2012. Grain iron and zinc association studies in rice (Oryza sativa L.) F1 progenies. Arch. Appl. Sci. Res. 4(1):696-702.

Ravindra Babu. V., Shreya. K. Kuldeep Singh Dangi., G. Usharani and P. Nagesh. 2012. Quantitative characters of bread wheat. Indus. J. Plant. Sci., 3: 189-192.

Renganathan, V. G., C. Vanniarajan, A. Nirmalakumari, M. Raveendran, S. Thiyageshwari and P. Arunachalam. 2017. Association Analysis in Germplasm and F2 Segregating Population of Barnyard Millet (Echinochloa frumentacea Roxb. Link) 
for Biometrical and Nutritional Traits. Int. J. Curr. Microbiol. App. Sci. 6(8): 3394-3400. doi: https://doi.org/ 10.20546/ijcmas.2017.608.406.

Sherif, E.A.A. 2007. Echinocloa colona (L.) Link: A promising species to cultivate salt affected soils in Arid lands. American-Eurasian J. Agric. Environ. Sci., 2 (6): 767-774.

Soman, R., N. Gande, R. Ambati, P. J. Kundur, A. R, Berhanu, D. Bekele and H. E. Shashidhar. 2014. Genetic variability and correlation studies for grain iron concentration and yield related traits in Recombinant Inbred Lines of rice (Oryza sativa L.) grown under aerobic condition. Int. J. Curr. Res., 6 (3), 5869-5874.
Suwarto, U. Susanto and S. Nurchasanah. 2015. Performance of selected plants in $F_{2}$ and $F_{3}$ generation for yield and yield component characters of new plant type rice genotypes at aerobic rice culture. Res. J. Pharm. Biol. Chem. Sci. 6 (1): 1165-1170.

Underwood, B.A. and S. Smitasiri. 2000. Micronutrient malnutrition: policies and programs for control and their implications. Annual review of nutrition. 19:303-324.

Vogel, K.P., F.A. Haskins and H.J. Gorz. 1980. Parent- progeny regression in Indian grass: inflation of heritability estimates by environmental covariances. Crop Sci., 20:580-582.

\section{How to cite this article:}

Renganathan, V.G. and Vanniarajan, C. 2018. Exploring the Barnyard Millet (Echinochloa frumentacea Roxb. Link) Segregating Population for Isolation of High Yielding, Iron and Zinc Content Genotype. Int.J.Curr.Microbiol.App.Sci. 7(04): 3611-3621. doi: https://doi.org/10.20546/ijcmas.2018.704.407 